\title{
Cardiac Masses
}

Although echocardiography is considered the primary technique for evaluating cardiac masses, magnetic resonance imaging (MRI) may be indicated in patients who have poor acoustic windows or in whom lesions are incompletely characterized by echocardiography. This unit presents the basic technique for evaluating cardiac masses, with optional contrast-enhanced sequences for specific indications. The parameters are based on experience on a Siemens 1.5 T Vision or Symphony and should be altered accordingly for different field strengths and manufacturers.

\section{IMAGING OF CARDIAC MASSES}

To image the heart, cardiac imaging packages that allow gated fast imaging of the heart using echo-train fast (or turbo) spin-echo and segmented $k$-space cine gradient-echo images are necessary for better imaging with examination times that are practical in the clinical setting.

Similar to that described for pericardial disease in UNIT A11.1, the core components of the imaging protocol are gated $T_{1}$-weighted and $T_{2}$-weighted turbo spin-echo images and cine gradient-echo images through the heart. The spin-echo images are used to provide high-resolution multiplanar imaging of the heart and to help to characterize masses. A fat-suppressed imaging sequence is optional for suspected germ cell tumors, lipomas, or lipomatous hypertrophy of the interatrial septum. The two-dimensional electrocardiogram (2-D ECG)-gated cine gradient-echo images provide views of a single slice at multiple phases of the cardiac cycle. When viewed in the cine mode, images can be used to evaluate the motion of the heart dynamically. Cine MRI can help distinguish a mass in a cardiac chamber from flowing blood and also is useful for evaluating physiologic effects of masses, such as mitral valvular obstruction by a left atrial myxoma. With fast breath-hold cine acquisitions, the total examination time for the Basic Protocol should be $<45$ min.

For patients with certain entities, such as thrombus versus mass or suspected intramural tumors, additional contrast-enhanced imaging of the heart can be performed by repeating $T_{1}$-weighted imaging following intravenous gadolinium contrast material.

Table A11.2.1 lists the hardware necessary to perform MRI of the heart. Higher gradient strengths with imaging sequences that allow gated turbo spin-echo and segmented cine gradient-echo imaging are preferable.

\section{Materials}

Normal saline $(0.9 \% \mathrm{NaCl})$, sterile

Gadolinium-based MR contrast agent (e.g., Magnevist, Omniscan, or Prohance)

Table A11.2.1 Equipment Parameters for Pericardial Imaging

\begin{tabular}{ll}
\hline Coil type & $\begin{array}{l}\text { Torso phased-array coil (or dedicated cardiac } \\
\text { coil, if available) }\end{array}$ \\
Gradient coil strength & $25-40 \mathrm{mT} / \mathrm{m}$ (or whatever the system permits) \\
Cardiac gating & Yes, preferably fiber-optic cables \\
Peripheral gating & No \\
Oxygen & Yes, 2 liters nasal cannula for most patients (to \\
& ensure breath-holding $>15 \mathrm{sec}$ ) \\
Use of contrast agents & Possible in patients with certain diseases (see \\
& Alternate Protocol) \\
\hline
\end{tabular}

Contributed by Vivian S. Lee

Current Protocols in Magnetic Resonance Imaging (2001) A11.2.1-A11.2.12

Copyright $\odot 2001$ by John Wiley \& Sons, Inc.
BASIC

PROTOCOL

Acquired Heart

Disease

A11.2.1 


\section{Set up equipment and patient}

1. As discussed in UNITA11.1 on pericardial disease, it is important to interview and screen the patient to ensure that he or she has no contraindications such as cardiac pacemaker or defibrillator or other ferromagnetic materials near vital structures. Also, ensure that the patient does not have health conditions, including those related to cardiac or pericardial disease, that may require emergency equipment during the scanning procedure.

Generally standard screening forms are used for all patients scanned in a magnetic resonance system.

The presence of any ferromagnetic metals may be a health hazard to the patient when he or she is inside the magnet and will also affect the imaging. If in doubt as to the exact composition of the items, it is best to exclude patients with any metal implants.

Patients may be accompanied into the magnet room by a friend or family member, who can sit in the room during the scan and comfort the patient as needed. This companion must be screened as well to ensure the absence of loose metal objects on the body or clothing.

2. If the procedure is a research protocol, have the patient sign any necessary consent form.

3. Have the patient remove all jewelry and change into a gown to eliminate any metal that might be found in clothing.

4. Inform the patient about what will occur during the procedure, what he or she will experience while in the magnet, and how to behave, including the following:

a. If earphones or headphones are used to protect the ears from the loud sounds produced by the gradients, ask the patient to wear these, but inform the patient that he or she will be able to communicate with you at any time during the imaging.

b. Give the patient a safety squeeze-bulb or similar equipment to request assistance at any time (demonstrate how this works).

c. For good results inform the patient not to talk and to avoid or minimize swallowing or other movement, during each scan-i.e., as long as the banging sounds continue.

Between scans, talking and swallowing are allowed in most cases but should be avoided when comparative positional studies are being performed; the patient will be informed when this is the case.

d. Inform the patient that, if necessary, he or she may call out at any time.

5. Inform the patient of the need to hold his or her breath (preferably at end expiration) for $\sim 15$ to $20 \mathrm{sec}$ for each cine gradient-echo acquisition. Assess need for supplementary oxygen to improve breath-holding capacity, and if necessary, administer 2 liters oxygen via nasal cannula. Advise the patient of the importance of not moving during the acquisition periods and of not taking deep breaths during the non-breath-hold acquisitions.

6. When the patient is on the table, place MRI-compatible ECG leads (Quatrode from In Vivo) according to manufacturer's guidelines. When possible, place leads on the back as this may reduce motion artifacts related to breathing. Make sure that the ECG tracing resulting from the lead placement results in high (positive) $R$ waves. If it does not, either reposition the leads or toggle the lead polarity to alternative options, if available on the system.

7. Place a pillow or other support under the knees to make the patient more comfortable. 
8. Place a $22-\mathrm{G}$ intravenous catheter in the antecubital fossa if the gadolinium contrast is deemed likely to be necessary.

A power injector can be used to administer contrast, but it is not necessary.

It is preferable to insert the line prior to imaging and to leave the patient in the magnet, with no intervening motion, between the scans run before contrast agent injection and those run after injection.

9. Center the patient's chest in the phased-array coil.

10. Use the centering light to position the patient and put him or her into the center of the magnet.

Once this step has been performed, so long as the patient does not move on the table, the table itself can be moved and then replaced in the same position as before without jeopardizing the positioning of one scan relative to another.

11. If the patient is unable to hold still, provide an appropriate sedative.

12. Once the patient has been centered in the magnet, check again to be sure that the ECG tracing demonstrates sharp positive $\mathrm{R}$ waves for suitable triggering. If not, then, with patient still positioned within the magnet, toggle lead polarity until a suitable tracing is obtained. If this is still not satisfactory, then bring the patient out of the magnet, check lead connections, and if necessary, reposition leads until a satisfactory tracing is obtained.

\section{Sequence 1: Rapid three-plane positioning scout}

13. For localization of subsequent acquisitions, run the system's three-plane scout scan (see Table A11.2.2). If possible, have the patient breath-hold at end expiration for the scout to better positioning of subsequent acquisitions.

\section{Sequence 2: 2-D transverse double inversion recovery half-Fourier single-shot turbo spin echo (db-HASTE)}

14. Use this ultrafast turbo spin-echo sequence (Table A11.2.3) with blood-nulling preparatory pulse for an overview of the heart to localize lesions for subsequent spin-echo imaging. Position a series of transverse slices off the scout images to ensure coverage of the entire heart.

This is a $T_{2}$-weighted sequence, and most tumors will appear high in signal intensity on HASTE.

The sequence can be performed with breath-holding at end expiration, but this is not absolutely necessary.

ECG-gating, also not necessary, can be used for improved images.

\section{Sequence 3: 2-D transverse $T_{1}$-weighted turbo spin-echo (TSE)}

15. Use the pilot scan and HASTE images to locate the mass in question or to define the extent of the cardiac abnormality. For greater coverage, run this sequence twice using the parameters shown in Table A11.2.4. If the area of coverage can be localized (e.g., for evaluation of a mass), only perform the sequence once with minimum slice gap (Fig. A11.2.1).

For $T_{1}$ weighting, $T_{R}$ should be $\sim 85 \%$ to $90 \%$ of the patient's $R$-to-R interval (time between $R$ waves) and $<900 \mathrm{msec}$. Short $T_{R}$ times will limit the number of slices that can be obtained in each acquisition.

16. Because acquisition times are too long to allow breath-hold imaging, with multiple acquisitions, instruct the patient to perform shallow breathing.

Acquired Heart Disease

A11.2.3 
Table A11.2.2 Primary Clinical Imaging Parameters for Sequence 1 (Pilot Scan)

Patient position

Scan type

Imaging plane (orientation)

Central slice or volume center

Echo time $\left(T_{\mathrm{E}}\right)$

Repeat time $\left(T_{\mathrm{R}}\right)$

Flip angle (FA)

Fields of view $\left(\mathrm{FOV}_{x}, \mathrm{FOV}_{y}\right)$

Resolution $(\Delta x, \Delta y)$

Number of data points collected $\left(N_{x}, N_{y}\right)$

Display matrix $\left(D_{x}, D_{y}\right)$

Slice thickness $(\Delta z)$

Number of slices

Slice gap

Number of acquisitions $\left(N_{\text {acq }}\right)$

Swap read and phase encoding

Saturation pulses

Scan time
Supine

Gradient echo

Three-plane (transverse, coronal, sagittal)

Center of chest

As short as possible

As short as possible

$15^{\circ}$

$500 \mathrm{~mm}, 500 \mathrm{~mm}$

$1.95 \mathrm{~mm}, 3.91 \mathrm{~mm}$

256,128

256,256

$10 \mathrm{~mm}$

5-10

$0-10$

1

No

Not applicable

5-15 sec

Table A11.2.3 Primary Clinical Imaging Parameters for Sequence 2 (HASTE)

Patient position

Scan type

Imaging plane (orientation)

Central slice or volume center

Echo time $\left(T_{\mathrm{E}}\right)$

Repeat time $\left(T_{\mathrm{R}}\right)$

Delay time $\left(T_{\mathrm{D}}\right)$

Flip angle (FA)

Fields of view $\left(\mathrm{FOV}_{x}, \mathrm{FOV}_{y}\right)$

Resolution $(\Delta x, \Delta y)$

Number of data points collected $\left(N_{x}, N_{y}\right)$

Display matrix $\left(D_{x}, D_{y}\right)$

Slice thickness $(\Delta z)$

Number of slices

Slice gap

Number of acquisitions $\left(N_{\text {acq }}\right)$

Swap read and phase encoding

Saturation pulses

ECG gating

Scan time
Supine

Single-shot fast spin echo

Transverse

Center of heart

43-63 msec (effective)

Infinity (echo spacing is $\sim 4 \mathrm{msec}$ )

$700 \mathrm{msec}$

$180^{\circ} a$

300-350 mm, 300r-350r mm, with $r=3 / 4$ (rectangular field of view) depending on body habitus 1.17-1.37 mm, 1.70-1.99 mm $256,176 r$, with $r=3 / 4$ (rectangular field of view) depending on body habitus

256, 256

5-8 mm

20

$0.8-2 \mathrm{~mm}$

1

No

No

Not necessary

20-30 sec

${ }^{a}$ The system displays the flip angle of the refocusing pulse. 
Table A11.2.4 Primary Clinical Imaging Parameters for Sequence 3 ( $T_{1}$-Weighted TSE)

\begin{tabular}{|c|c|}
\hline Patient position & Supine \\
\hline Scan type & Turbo spin echo \\
\hline Imaging plane (orientation) & Transverse \\
\hline Central slice or volume center & Center of heart \\
\hline Echo time $\left(T_{\mathrm{E}}\right)$ & $12 \mathrm{msec}$ (or minimum) \\
\hline Echo train length (ETL) & 3 \\
\hline Repeat time $\left(T_{\mathrm{R}}\right)$ & $\begin{array}{l}<900 \text { msec (and }<90 \% \text { of the } \\
\text { R-to-R interval) }\end{array}$ \\
\hline Delay time after $\mathrm{R}$ wave & $0 \mathrm{msec}$ \\
\hline Flip angle (FA) & $180^{\circ a}$ \\
\hline Fields of view $\left(\mathrm{FOV}_{x}, \mathrm{FOV}_{y}\right)$ & $\begin{array}{l}300-350 \mathrm{~mm}, 300 r-350 r \mathrm{~mm} \text {, } \\
\text { with } r=3 / 4 \text { (rectangular field of } \\
\text { view) depending on body habitus }\end{array}$ \\
\hline Resolution $(\Delta x, \Delta y)$ & $1.17-1.37 \mathrm{~mm}, 1.43-1.67 \mathrm{~mm}$ \\
\hline Number of data points collected $\left(N_{x}, N_{y}\right)$ & $\begin{array}{l}256,210 r, \text { with } r=3 / 4 \\
\text { (rectangular field of view) } \\
\text { depending on body habitus }\end{array}$ \\
\hline Display matrix $\left(D_{x}, D_{y}\right)$ & 256,256 \\
\hline Slice thickness $(\Delta z)$ & 4-8 mm \\
\hline Number of slices & $10-12$ \\
\hline Slice gap & $\begin{array}{l}0.4 \text { to } 0.8 \mathrm{~mm} \text { or } 4-8 \mathrm{~mm} \text { if } \\
\text { concatenated }^{b}\end{array}$ \\
\hline Number of acquisitions $\left(N_{\text {acq }}\right)$ & $3-5$ \\
\hline Swap read and phase encoding & No \\
\hline Saturation pulses & No \\
\hline ECG gating & Yes \\
\hline Scan time & $3-8 \min$ \\
\hline \multicolumn{2}{|c|}{$\begin{array}{l}{ }^{a} \text { The system displays the flip angle of the refocusing pulse. The flip angle of the first pulse of this } \\
\text { sequence is } 90^{\circ} \text {. } \\
{ }^{b} \text { If the } z \text {-axis coverage needed is on the order of } 60 \text { to } 80 \mathrm{~mm} \text {, then a single acquisition with about } 8 \\
\text { to } 10 \text { slices at } 6 \text { to } 8 \mathrm{~mm} \text { thickness and minimal gap }(0.6 \text { to } 0.8 \mathrm{~mm} \text { ) can be performed. For complete } \\
\text { coverage of the chest, two separate acquisitions of } 8 \text { to } 10 \text { slices can be performed, each with a gap } \\
\text { equal to the slice thickness (e.g., } 8 \mathrm{~mm} \text { slice thickness with } 8 \mathrm{~mm} \text { gap). In this example, the second } \\
\text { acquisition should be performed with the slice positions shifted by } 8 \mathrm{~mm} \text { and with the same thickness } \\
\text { and gap, so that with the two acquisitions together, complete coverage of the chest will be obtained } \\
\text { with } 8 \text {-mm-thick slices and effectively no gap. }\end{array}$} \\
\hline
\end{tabular}

\section{Sequence 4: 2-D fat-suppressed transverse $T_{2}$-weighted inversion-recovery turbo spin echo (optional)}

17. Perform limited transverse $T_{2}$-weighted turbo spin-echo images through the heart as needed.

An inversion-recovery technique will provide useful fat suppression and allow characterization of fatty masses, such as lipomas of the heart (Fig. A11.2.2).

18. Set the imaging parameters as shown in Table A11.2.5 to obtain a single slice per breath-hold. Instruct the patient to breath-hold at end expiration, if possible, for best reproducibility.

The $T_{R}$ plus delay time should be $<85 \%$ to $90 \%$ of two R-to-R intervals.

19. Adjust the inversion time depending on the particular manufacturer. Select delay times to allow imaging in diastole.

The inversion time determines the degree of fat suppression obtained.

Acquired Heart

Disease

A11.2.5 


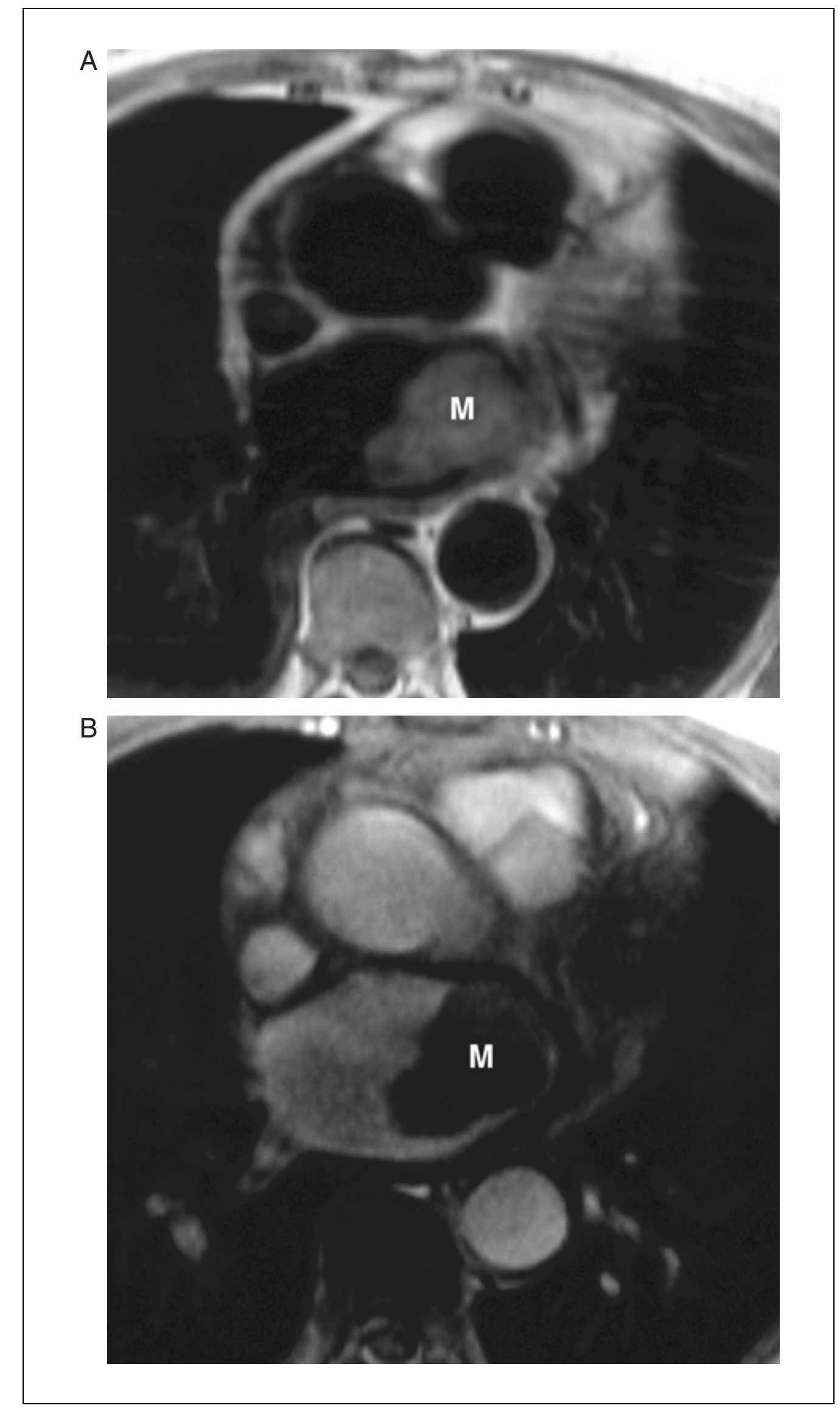

Figure A11.2.1 Left atrial myxoma. (A) Transverse $T_{1}$-weighted turbo spin echo and (B) transverse cine gradient echo show a large mass in the left atrium (M). 


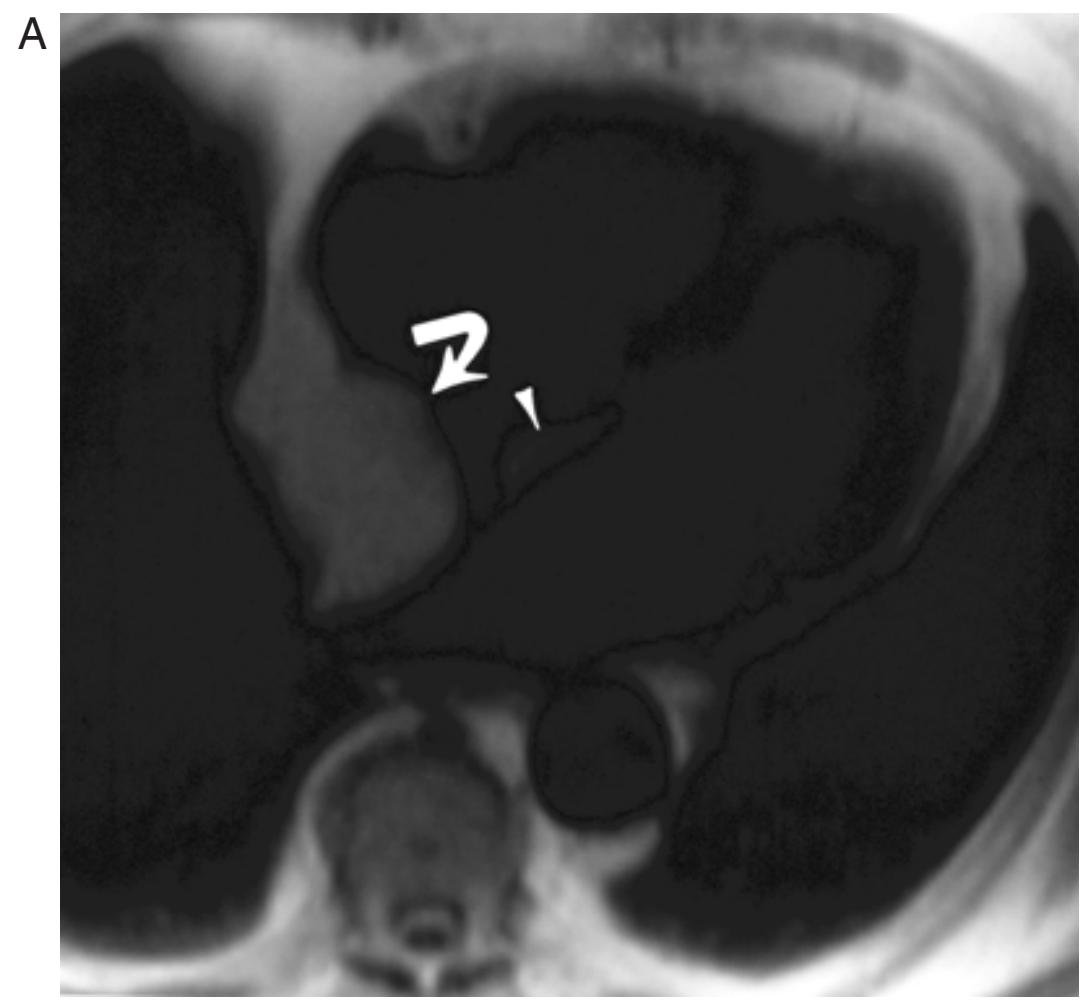

B

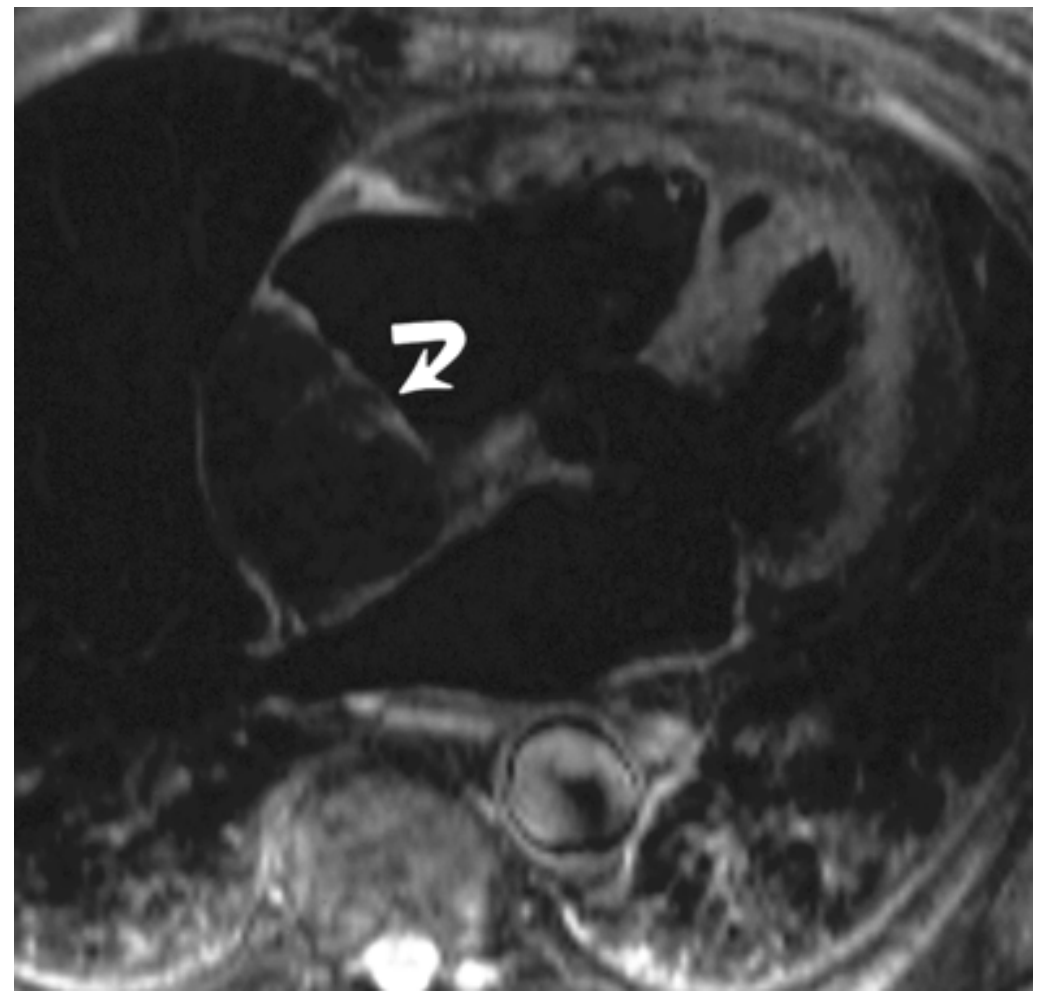

Figure A11.2.2 Atrial lipoma. (A) Transverse HASTE image and (B) transverse short $T_{1}$ inversionrecovery image through the right atrium demonstrate a large mass in the right atrial wall (curved arrows) and also involving the interatrial septum (shorter arrowhead in A). The lesion is high in signal intensity on the HASTE image. The loss of signal intensity in the mass on the inversion-recovery sequence parallels that of subcutaneous fat and supports the diagnosis of right atrial lipoma with involvement of the internal septum. 
Table A11.2.5 Primary Clinical Imaging Parameters for Sequence 4 (Short Tau Inversion Recovery, Optional)

\begin{tabular}{|c|c|}
\hline Patient position & Supine \\
\hline Scan type & Inversion recovery fast spin echo \\
\hline Imaging plane (orientation) & Transverse \\
\hline Central slice or volume center & Center of heart \\
\hline Echo time $\left(T_{\mathrm{E}}\right)$ & $57-76 \mathrm{msec}$ \\
\hline Echo train length (ETL) & 33 \\
\hline Repeat time $\left(T_{\mathrm{R}}\right)$ & $\begin{array}{l}<900 \text { msec }\left[T_{\mathrm{R}}+T_{\mathrm{D}} \text { should be }<2\right. \\
\times(85 \% \text { to } 90 \%) \times(\mathrm{R} \text {-to- } \mathrm{R} \\
\text { interval })]\end{array}$ \\
\hline Inversion time $\left(T_{\mathrm{I}}\right)$ & $170 \mathrm{msec}$ \\
\hline Delay time $\left(T_{\mathrm{D}}\right)$ & $500 \mathrm{msec}$ \\
\hline Flip angle (FA) & $180^{\circ}$ \\
\hline Fields of view $\left(\mathrm{FOV}_{x}, \mathrm{FOV}_{y}\right)$ & $\begin{array}{l}300-350 \mathrm{~mm}, 300 r-350 r \mathrm{~mm} \text {, } \\
\text { with } r=3 / 4 \text { (rectangular field of } \\
\text { view) depending on body habitus }\end{array}$ \\
\hline Resolution $(\Delta x, \Delta y)$ & $1.17-1.37 \mathrm{~mm}, 1.67-1.94 \mathrm{~mm}$ \\
\hline Number of data points collected $\left(N_{x}, N_{y}\right)$ & $\begin{array}{l}256,180 r, \text { with } r=3 / 4 \\
\text { (rectangular field of view) } \\
\text { depending on body habitus }\end{array}$ \\
\hline Display matrix $\left(D_{x}, D_{y}\right)$ & 256,256 \\
\hline Slice thickness $(\Delta z)$ & $8 \mathrm{~mm}$ \\
\hline Number of slices & 1 \\
\hline Slice gap & Not applicable \\
\hline Number of acquisitions $\left(N_{\mathrm{acq}}\right)$ & 1 \\
\hline Swap read and phase encoding & No \\
\hline Saturation pulses & No \\
\hline ECG gating & Yes \\
\hline Scan time & $12-15$ \\
\hline
\end{tabular}

\section{Sequence 5: 2-D transverse and coronal fast cine gradient echo}

20. Generally, perform transverse cine gradient-echo imaging, slice by slice, through the entire heart. Obtain additional coronal or sagittal oblique views for evaluation of specific regions, for example, through the inferior vena cava for evaluation of vascular extension of tumor thrombus from hepatoma or renal cell carcinoma.

Cine gradient-echo images can be performed selectively. A four-chamber view or twochamber view of the heart may be useful to determine whether an atrial mass interferes with mitral or tricuspid valvular function.

21. Set the imaging parameters as shown in Table A11.2.6 to obtain a single slice per breath-hold. Adjust the parameters depending on the patient's breath-holding capability and heart rate. For patients with slower heart rates, use sequences that provide a greater number of lines per segment to help reduce acquisition times. Set the number of phases of the cardiac study according to the formula:

Number of cardiac phases $=($ R-to-R interval $) \times 85 \% / T_{\mathrm{R}}$

Parameters shown in Table A11.2.6 are for a segmented $k$-space approach with nine lines of $k$-space per segment and four echoes shared.

22. Instruct the patient to breath-hold at end expiration, if possible, for best reproducibility. If patient is unable to breath-hold, perform the sequence with multiple 
Table A11.2.6 Primary Clinical Imaging Parameters for Sequence 5 (Cine Gradient Echo)

\begin{tabular}{|c|c|}
\hline Patient position & Supine \\
\hline Scan type & $\begin{array}{l}\text { Segmented } k \text {-space cine gradient } \\
\text { echo }\end{array}$ \\
\hline Imaging plane (orientation) & Transverse (coronal is optional) \\
\hline Central slice or volume center & Center of heart \\
\hline Echo time $\left(T_{\mathrm{E}}\right)$ & $4.8 \mathrm{msec}$ (or minimum) \\
\hline Number of lines per segment & 9 (see step 21) \\
\hline Repeat time $\left(T_{\mathrm{R}}\right)$ & 80-100 msec (temporal resolution) \\
\hline Delay time $\left(T_{\mathrm{D}}\right)$ & $0 \mathrm{msec}$ \\
\hline Flip angle (FA) & $20^{\circ}$ \\
\hline Fields of view $\left(\mathrm{FOV}_{x}, \mathrm{FOV}_{y}\right)$ & $\begin{array}{l}300-350 \mathrm{~mm}, 300 r-350 r \mathrm{~mm} \text {, } \\
\text { with } r=3 / 4 \text { (rectangular field of } \\
\text { view) depending on body habitus }\end{array}$ \\
\hline Resolution $(\Delta x, \Delta y)$ & $1.17-1.37 \mathrm{~mm}, 1.70-1.99 \mathrm{~mm}$ \\
\hline Number of data points collected $\left(N_{x}, N_{y}\right)$ & $\begin{array}{l}256,176 r, \text { with } r=3 / 4 \\
\text { (rectangular field of view) } \\
\text { depending on body habitus }\end{array}$ \\
\hline Display matrix $\left(D_{x}, D_{y}\right)$ & 256,256 \\
\hline Slice thickness & $5-7 \mathrm{~mm}$ \\
\hline Number of slices & 1 \\
\hline Slice gap & Not applicable \\
\hline Number of acquisitions $\left(N_{\text {acq }}\right)$ & 1 \\
\hline Swap read and phase encoding & No \\
\hline Saturation pulses & No \\
\hline Number of cardiac phases & $(\mathrm{R}$-to-R interval $) \times 85 \% / T_{\mathrm{R}}$ \\
\hline ECG gating & Yes \\
\hline Scan time & $15-20 \mathrm{sec}$ \\
\hline
\end{tabular}

acquisitions (three-signal averages, for example) with the patient breathing normally or shallowly.

\section{Sequence 6: 2-D transverse $T_{1}$-weighted turbo spin echo (optional)}

For patients with specific clinical indications, such as the differentiation between cardiac tumor and thrombus or suspected intramural masses, contrast-enhanced images may also be needed.

23. Leaving the patient in the magnet, inject the contrast agent, flush the line with $20 \mathrm{ml}$ saline.

Intravenous gadolinium contrast material at a dose of $0.1 \mathrm{mmol} / \mathrm{kg}$ is administered and turbo $T_{1}$-weighted spin-echo images repeated. Non-enhanced images can be subtracted from contrast-enhanced images using post-processing software to depict enhancing lesions.

24. Repeat the sequence described in Table A11.2.4, ensuring that all imaging parameters are kept the same to facilitate post-processing.

\section{COMMENTARY}

\section{Background Information}

The primary screening test for suspected intracardiac masses is transthoracic echocardiography. However, there are limitations with this technique. The limited field of view, dependence on adequate acoustic window and skill of the echocardiographer, and limited visualization of certain areas of the heart such
Acquired Heart

Disease

A11.2.9 
as the apex or left atrial appendage require additional performance of transesophageal echocardiography in many cases, which is invasive and also limited in field of view (Martin et al., 1998). Magnetic resonance imaging can provide a noninvasive yet comprehensive anatomic and physiologic assessment of cardiac masses by relying on spin-echo imaging for anatomy and cine gradient-echo imaging for flow and function.

The most common cardiac mass is thrombus, which typically lies in a dilated left atrium or ventricle. Thrombus can usually be distinguished from most neoplastic masses by morphology and the lack of contrast enhancement. Benign tumors include myxomas, typically arising in the left atrium with a pedicle attached to the atrial septum, and lipomas, usually in the right atrium as a discrete mass or more commonly a uniform fatty infiltration of the interatrial septum (Fig. A11.2.2). Lipomas can be characterized by using fat suppression sequences to identify the presence of fat within lesions. In children, rhabdomyomas can be found, typically within the ventricles, and are associated with tuberous sclerosis. Malignant tumors are most commonly secondary, such as direct extension from adjacent lung or mediastinal malignancies, vascular extension from renal cell carcinoma or hepatoma, or hematogenous metastases, such as melanoma or sarcoma. Primary cardiac malignancies are extremely rare, the most common being angiosarcoma (Higgins, 1992). Unlike benign tumors, malignancies of the heart typically are less well-circumscribed and demonstrate aggressive infiltrative appearance, best seen on contrast-enhanced images. Magnetic resonance imaging can also help to differentiate cardiac masses from adjacent paracardiac pathology (Barakos et al., 1989).

\section{Critical Parameters and Troubleshooting}

\section{ECG tracings}

As discussed in UNITAII.I on pericardial imaging, ECG tracings are vital for all imaging of the heart. Sharp up-going $\mathrm{R}$ waves with significantly smaller (or inverted) $\mathrm{T}$ waves are needed since triggering typically depends on the ECG value crossing a particular threshold. Magnetic resonance-compatible ECG leads with conductive gel should be used. For good skin contact, the patient's skin may need to be shaved. The position of the heart and axes of the conduction pathways may vary considerably across patients. The manufacturer's recommendations of lead positioning should be used as a starting point. If polarity is not as desired (e.g., inverted $\mathrm{R}$ waves), check that leads are positioned correctly (RA to the right side, LA to the left side, etc), and then, if needed, toggle through the lead polarity options (I, II, AVR, etc.). Finally, as a last resort, reposition ECG leads, using new adhesive pads to ensure adequate adhesion and conduction gel. If ECG tracings are lost during positioning of the patient or scanning, check the patient to ensure that leads have remained properly connected.

\section{Customization of gated MRI parameters}

Magnetic resonance imaging of the heart and pericardium requires careful customization of imaging parameters according to patient heart rate and breath-holding capability (see UNIT Al1.l on pericardial imaging). For a multislice turbo spin-echo sequence, the number of slices that can be imaged in one acquisition depends on the $T_{\mathrm{R}}$, which in turn depends on the patient's heart rate. For single-slice cine gradient-echo images, multiple phases of the heart cycle are imaged at a single slice position. The more lines of $k$ space (number of phase-encoding lines) that are collected for each cardiac phase during each $T_{\mathrm{R}}$ interval, the faster the acquisition time, but fewer cardiac phases can be acquired. While sequences could be customized for every patient, to simplify the process, system protocols should be set up for specific categories of patients, such as "slow heart rate" and "fast heart rate" with the aim of limiting acquisition times $<20 \mathrm{sec}$.

On some systems, a view-sharing option will be available for cine gradient-echo sequences that enables almost double or greater temporal resolution (number of cardiac phases) in the same acquisition time.

\section{Motion or breathing artifacts}

Cine gradient-echo images can be performed with multiple signal averages when patients are unable to breath-hold adequately. Supplemental oxygen via nasal cannula can improve a patient's breath-holding capability substantially and should be considered in all patients.

\section{Imaging infants and children}

MR imaging techniques must be modified for the evaluation of suspected masses in infants and children. A head coil should be used for small infants. Non-breath-hold modifications such as multiple signal averages should be used. 


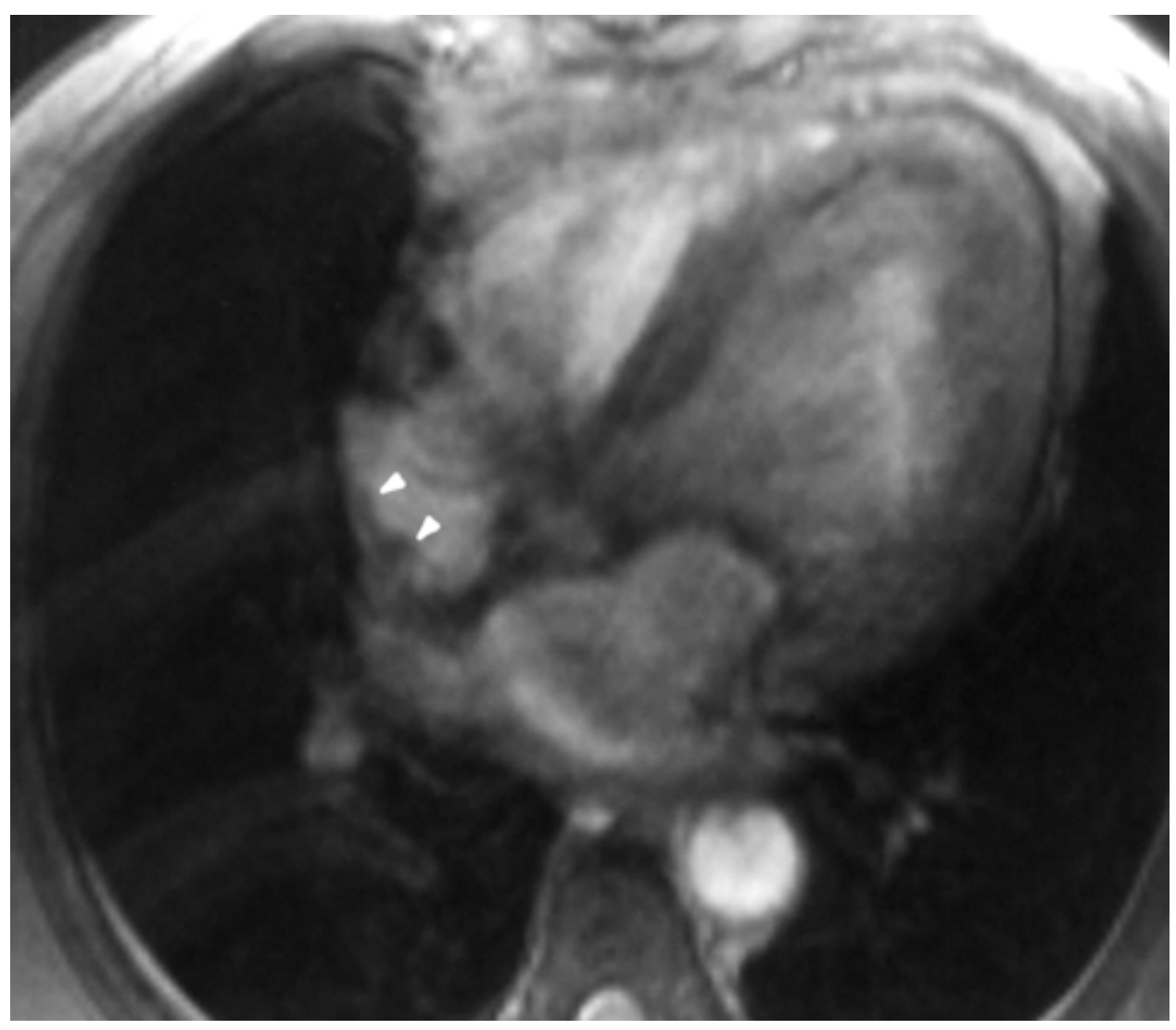

Figure A11.2.3 Right atrial pseudotumor. Transverse cine gradient-echo image shows a typical nodular appearance of the right atrial pseudotumor, seen along the posterior wall of the right atrium (arrowheads). This is a normal structure and should not be mistaken for tumor or thrombus.

Appropriate sedation should be administered when necessary.

\section{Pseudotumors}

In $\sim 59 \%$ to $90 \%$ of patients, a nodular or linear density can be seen along the posterior wall of the right atrium (Mirowitz and Gutierrez, 1992; Meier and Hartnell, 1994). This characteristic lesion can be misinterpreted as an intracardiac mass and cause potential diagnostic difficulties if unrecognized. The mass is caused by normal fibromuscular elements of the right atrium, such as the crista terminalis and Chiari network (Fig. A11.2.3).

\section{Anticipated Results}

The goal of MRI of the cardiac masses is to localize and characterize as best possible masses within the heart. With adequate ECG gating, MRI can provide clear visualization of anatomic abnormalities (Fig. A11.2.1) as well as an assessment of consequent hemodynamic abnormalities. Magnetic resonance imaging can be used to characterize lipomas and lipomatous hypertrophy of the interatrial septum
(Fig. A11.2.2). Vascular or adjacent extension of tumor into the heart can be visualized because of the large field of view available with MRI.

Contrast material can supplement cine gradient-echo imaging in helping to differentiate nonenhancing masses such as thrombus from neoplastic disease. However, in many cases, contrast material is not needed to diagnose and characterize cardiac masses.

\section{Literature Cited}

Barakos, J.A., Brown, J.J., and Higgins, C.B. 1989. MR imaging of secondary cardiac and paracardiac lesions. Am. J. Roentgenol. 153:47-50.

Higgins, C.B. 1992. Essentials of Cardiac Radiology and Imaging. J.B. Lippincott, New York.

Martin, E.T., Fuisz, A.R., and Pohost, G.M. 1998. Imaging cardiac structure and pump function. Cardiol. Clin. 16:135-160

Meier, R.A. and Hartnell, G.G. 1994. MRI of right atrial pseudomass: Is it really a diagnostic problem? J. Comput. Assist. Tomogr. 18:398-401.

Mirowitz, S.A. and Gutierrez, F.R. 1992. Fibromuscular elements of the right atrium: Pseudomass at MR imaging. Radiology 182:231-233.
Acquired Heart

Disease 


\section{Key References}

Dupuis, K., Thangaraj, V., and Edelman, R.R. 1996. Practical MRI for the technologist and imaging specialist. In Clinical Magnetic Resonance Imaging (R.R. Edelman, J.R. Hesselink, and M.B. Zlatkin, eds.) pp. 52-87. W.B. Saunders, Philadelphia.

Covers a wide range of practical information for setting patients up for MRI and also includes a useful description of cardiac imaging sequence parameters.

Higgins, 1992. See above.
Contains a detailed description of methods to evaluate cardiac masses, including plain film diagnoses and MRI.

White, C.S. 1996. MR evaluation of the pericardium and cardiac malignancies. MRI Clin. North Am. 4:237-251.

Reviews MRI findings of cardiac masses.

Contributed by Vivian S. Lee

New York University Medical Center

New York, New York 